\title{
Características Produtivas e Reprodutivas de Fêmeas da Raça Nelore e Cruzadas 1/2 Canchim + 1/2 Nelore
}

\author{
Maurício Mello de Alencar ${ }^{1,2}$, Rogério Taveira Barbosa1, Antônio Pereira de Novaes ${ }^{1}$
}

\begin{abstract}
RESUMO - O objetivo deste estudo foi avaliar os efeitos de fatores de meio e genéticos sobre o peso ao parto (PVP) de vacas Nelore e 1/2 Canchim + 1/2 Nelore, acasaladas com touros das raças Canchim e Charolesa, respectivamente, e também sobre o período de gestação (PG) de seus bezerros e sobre os pesos $(\mathrm{P})$ e as idades (I) à primeira inseminação (PI), primeira concepção (PC) e primeira parição (PP) das novilhas $1 / 2$ Canchim $+1 / 2$ Nelore. O método dos quadrados mínimos foi usado com modelos matemáticos que incluíram os efeitos de ano e mês do parto, idade da vaca ao parto e origem da vaca (para as vacas Nelore) para a análise de PVP, estação de monta, idade da vaca, origem da vaca, sexo do bezerro e os efeitos aleatórios de pai do bezerro, para a análise de PG, e ano e época de nascimento e a interação ano x época, para as características das novilhas. Ano do parto e origem da vaca apresentaram efeito sobre PVP. As vacas paridas de agosto a novembro foram mais leves ao parto que as paridas em dezembro e de maio a julho. O peso ao parto aumentou linearmente com a idade da vaca. O PG dos machos foi maior que o das fêmeas, para os bezerros $1 / 2$ Canchim + $1 / 2$ Nelore. As médias estimadas de PVP foram iguais a $450 \pm 2 \mathrm{~kg}$ (vacas Nelore) e $502 \pm 2 \mathrm{~kg}$ (vacas $1 / 2 \mathrm{Canchim}+1 / 2 \mathrm{Nelore}$ ) e as de PG, 291,0 \pm 0,4 dias (bezerros 1/2 Canchim $+1 / 2$ Nelore) e 285,7 \pm 0,2 dias (bezerros 1/2 Charolês $+1 / 4$ Canchim $+1 / 4$ Nelore) Para as novilhas cruzadas, as médias estimadas foram iguais a $372 \pm 2 \mathrm{~kg}$ (PPI), $376 \pm 2 \mathrm{~kg}$ (PPC), $442 \pm 3 \mathrm{~kg}$ (PPP), $810 \pm 5$ dias (IPI), $831 \pm 6$

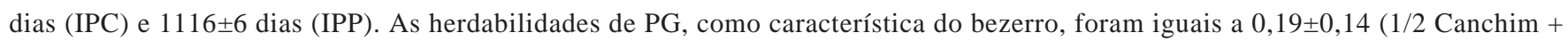
$1 / 2$ Nelore ) e $0,69 \pm 0,31(1 / 2$ Charolês $+1 / 4$ Canchim $+1 / 4$ Nelore $)$.
\end{abstract}

Palavras-chave: bovinos de corte, fêmeas, fertilidade, herdabilidade, pesos

\section{Productive and Reproductive Traits of Straightbred Nellore and Crossbred 1/2 Canchim + 1/2 Nellore Females}

ABSTRACT - The objective of this study was to evaluate the effects of genetic and non genetic factors on cow weight at calving (CWC) of Nellore and 1/2 Canchim $+1 / 2$ Nellore cows, mated to Canchim (5/8 Charolais $+3 / 8$ Zebu) and Charolais breed bulls, respectively, and also on the gestation length (GL) of their calves, and the weights (W) and ages (A) at first insemination (FI), conception (FCo), and calving (FC) of 1/2 Canchim + 1/2 Nellore heifers. The least squares method was used, with models that included the effects of year and month of calving, age of cow and origin of cow (for the Nellore cows only) for the analyses of CWC, breeding season, age of cow, origin of cow and sire of calf (random) for the analyses of GL, and year and season of birth and the interaction year x season for the analyses of the traits of the heifers. Year of calving and origin of cow affected CWC. The cows calving from August to November were lighter than the cows calving in December and from May to July. Cow weight linearly increased with age of cow. For the crossbred Canchim x Nellore calves, gestation length was longer for males than for females. The CWC least square means were $450 \pm 2 \mathrm{~kg}(\mathrm{Nellore}$ cows $)$ and $502 \pm 2 \mathrm{~kg}(1 / 2$ Canchim $+1 / 2$ Nellore cows $)$ and the GL least square means were $291.0 \pm .4$ days $(1 / 2$ Canchim $+1 / 2$ Nellore calves $)$ and $285.7 \pm .2$ days (1/2 Charolais $+1 / 4$ Canchim $+1 / 4$ Nellore calves $)$. The least square means were $372 \pm 2 \mathrm{~kg}$ (WFI), 376 $2 \mathrm{~kg}$ (WFCo), 442 $\pm 3 \mathrm{~kg}$ (WFC), $810 \pm 5$ days (AFI), 831 \pm 6 days (AFCo), and $1116 \pm 6 \mathrm{days}$ (AFC) for the $1 / 2$ Canchim $+1 / 2$ Nellore heifers. The heritabilities of GL, as a trait of the calf, were equal to $.17 \pm .14(1 / 2 \mathrm{Canchim}+1 / 2 \mathrm{Nellore})$ and $.69 \pm .31(1 / 2$ Charolais $+1 / 4$ Canchim $+1 / 4$ Nellore $)$.

Key Words: beef cattle, females, fertility, heritabilities, weights

\section{Introdução}

As diferenças genéticas existentes entre as raças bovinas podem ser utilizadas, no sentido de adequar tipo de animal e ambiente, para aumentar a eficiência dos sistemas de produção (DICKERSON, 1969). O cruzamento entre raças e a seleção dentro de raças são métodos utilizados para aproveitar essas diferenças. Neste contexto, o conhecimento do potencial produtivo de diferentes grupos genéticos e de fatores de meio e genéticos que influenciam características de importância econômica é fundamental no deline- 
amento de programas de manejo, seleção e cruzamento.

As características econômicas mais importantes na produção de bovinos de corte são a eficiência reprodutiva do rebanho e a taxa de crescimento dos animais (WILHAM, 1971). Entre as características reprodutivas estão aquelas ligadas à precocidade das fêmeas, como as idades à primeira concepção e ao primeiro parto. Essas características têm sido objeto de estudo no Brasil, entretanto, na sua grande maioria, com animais "puros" de raças bovinas de corte (OLIVEIRA FILHO et al., 1975; MARIANTE, 1978; BALIEIRO et al., 1980; MIRANDA et al., 1982, ALENCAR e BUGNER, 1986; e 1987).

O período de gestação é uma característica que exerce influência sobre a produtividade dos rebanhos, pois está associado ao peso ao nascimento (REYNOLDS et al., 1980) e à data do parto e ao intervalo de partos (BOURDON e BRINKS, 1983). Segundo WRAY et al. (1987), a redução no período de gestação, além de reduzir problemas de parto, pode aumentar o intervalo pós-parto até a estação de monta, proporcionando mais tempo às vacas para retornarem ao cio. No Brasil, são poucos os trabalhos de pesquisa sobre o período de gestação em bovinos de corte (SILVA et al., 1979; SILVA e PEREIRA, 1986; ALENCAR e BUGNER, 1987, ALENCAR et al., 1996; e SCARPATI, 1997).

O peso da vaca é outra característica que deve ser considerada, uma vez que a manutenção do rebanho de vacas é fator importante na eficiência econômica dos sistemas de produção. Vacas mais pesadas e que produzem mais leite consomem mais alimentos (McMORRIS e WILTON, 1986) e podem não ser as mais eficientes (EUCLIDES FILHO et al., 1995). No Brasil, alguns autores trabalharam com essa característica em bovinos de corte (BARBOSA, 1986; PÁDUA et al., 1994; e ALENCAR et al., 1997a).

A Associação Brasileira de Criadores de Canchim (ABCCAN) permite quatro diferentes sistemas de acasalamentos para sechegaraobovinobimestiço Canchim (5/8 Charolês + 3/8 Zebu). Um desses sistemas envolve o acasalamento de fêmeas zebuínas com touros Canchim para produzir fêmeas 1/2 Canchim $+1 / 2$ Zebu, que cruzadas com touros Charolês produzem machos e fêmeas 1/2 Charolês + 1/4 Canchim + 1/4 Zebu, que acasalados entre si produzem o bovino Canchim.

O objetivo deste trabalho foi avaliar o efeito de fatores genéticos e não-genéticos sobre as idades e os pesos à primeira inseminação, à primeira concepção e ao primeiro parto de fêmeas $1 / 2$ Canchim + $1 / 2$ Nelore, peso ao parto de fêmeas Nelore e
1/2 Canchim $+1 / 2$ Nelore e período de gestação de bezerros $1 / 2$ Canchim $+1 / 2$ Nelore e $1 / 2$ Charolês + 1/4 Canchim + 1/4 Nelore.

\section{Material e Métodos}

Foram utilizados dados de dois arquivos de animais pertencentes ao rebanho de bovinos de corte do Centro de Pesquisa de Pecuária do Sudeste (CPPSE) da Empresa Brasileira de Pesquisa Agropecuária (EMBRAPA), situado no município de São Carlos, Estado de São Paulo. O primeiro arquivo é constituído por observações de fêmeas da raça Nelore acasaladas com touros da raça Canchim, para produzir animais $1 / 2$ Canchim $+1 / 2$ Nelore. O segundo arquivo é composto de observações de fêmeas $1 / 2$ Canchim + $1 / 2$ Nelore inseminadas com sêmen de touros da raça Charolesa, para produzir animais 1/2 Charolês + 1/4 Canchim + 1/4 Nelore. Os animais deste estudo pertencem a um projeto de obtenção de novas linhagens de bovinos da raça Canchim. Os animais cruzados 1/2 Canchim $+1 / 2$ Nelore são filhos de uma amostra grande de touros Canchim da própria Embrapa. Os animais 1/2 Charolês + 1/4 Canchim + 1/4 Nelore são filhos de nove touros da raça Charolesa, de linhagens diferentes, cujo sêmen foi importado. As vacas da raça Nelore pertenciam a cinco rebanhos (origem) diferentes. As vacas Nelore de origem um a quatro foram adquiridas de três propriedades em diferentes pontos do Estado de São Paulo e de uma propriedade no Estado de Mato Grosso do Sul, com idades variando de bezerra à vaca adulta. Apenas os animais da origem cinco nasceram na fazenda do CPPSE. Todos os animais do rebanho foram mantidos em regime exclusivo de pastos dos capins braquiária (Brachiaria decumbens) e andropogon (Andropogon gayanus), recebendo suplemento mineral à vontade durante todo o ano. As estações de monta normalmente tiveram início em agosto e término em dezembro; entretanto, em alguns anos, tiveram início mais ao final do ano e término no início do ano seguinte. Em 1991, também existiu uma estação de monta de meio de ano (maio a julho). As novilhas entravam em reprodução, em geral, próximo dos dois anos de idade. Os touros da raça Canchim portavam buçal marcador e foram colocados individualmente com, aproximadamente, 30 vacas Nelore. No caso das fêmeas 1/2 Canchim $+1 / 2$ Nelore, a detecção de cio foi feita com a ajuda de rufiões que também portavam buçal marcador. Os lotes de reprodução foram vistoriados duas vezes ao dia para as anota- 
962 Rev. bras. zootec.

ções de cobertura e detecção do cio. Todas as fêmeas foram pesadas logo após o parto (até 24 horas após) e as fêmeas 1/2 Canchim $+1 / 2$ Nelore, no momento da inseminação.

As observações consideradas neste estudo foram 631 pesos ao parto (PVP) e 440 períodos de gestação (PG) de 169 vacas da raça Nelore, 219 pesos (PPI) e 220 idades (IPI) à primeira inseminação, 205 pesos (PPC) e 206 idades (IPC) à primeira concepção, e 209 pesos (PPP) e idades (IPP) ao primeiro parto de novilhas $1 / 2$ Canchim + 1/2 Nelore, 628 pesos ao parto e 503 períodos de gestação de 213 e 208 vacas $1 / 2$ Canchim $+1 / 2$ Nelore, respectivamente.

As observações foram analisadas pelo método dos quadrados mínimos, utilizando-se o procedimento GLM (SAS, 1996). Os modelos matemáticos utilizados para avaliar o peso ao parto das vacas Nelore e cruzadas 1/2 Canchim $+1 / 2$ Nelore incluíram os efeitos de ano (1986 a 1994 para as Nelore e 1991 a 1997 para as 1/2 Canchim $+1 / 2$ Nelore) e mês (maio a dezembro) do parto, origem da vaca Nelore e idade da vaca ao parto ( $4 \mathrm{a} \geq 11$ anos para as Nelore, e $3 \mathrm{a} \geq 9$ anos para as $1 / 2$ Canchim $+1 / 2$ Nelore). Os modelos para analisar o período de gestação dos bezerros incluíram os efeitos de estação de monta (1985 a 1993 para os 1/2 Canchim + 1/2 Nelore e 1989 a 1996 para os $1 / 2$ Charolês $+1 / 2$ Canchim $+1 / 2$ Nelore), sexo do bezerro, origem da vaca Nelore e idade da vaca ao parto, além dos efeitos aleatórios de pai do bezerro aninhado dentro de estação de monta, para os bezerros cruzados Canchim, e pai do bezerro, para os bezerros cruzados Charolês. Os modelos matemáticos utilizados para analisar as observações das novilhas 1/2 Canchim + 1/2 Nelore incluíram os efeitos de ano (1987 a 1994) e época (maio a agosto e setembro a dezembro) de nascimento da novilha e da interação ano x época. Para estimar a herdabilidade do período de gestação, como característica do bezerro, utilizouse o componente de variância do pai do bezerro e obteve-se o erro-padrão da estimativa, segundo SWIGER et al. (1964). Considerou-se o período de gestação como característica do bezerro, e não como característica da vaca, uma vez que WRAY et al. (1987) verificaram maior importância relativa do efeito aditivo direto que do efeito aditivo materno sobre o período de gestação, indicando que o período de gestação é determinado mais pelo bezerro que pela mãe.

\section{Resultados e Discussão}

Os resumos das análises de variância dos pesos das vacas ao parto e dos períodos de gestação são apresentados na Tabela 1. Observa-se que o ano do parto influenciou $(\mathrm{P}<0,01)$ o peso ao parto das fêmeas Nelore e 1/2 Canchim + 1/2 Nelore. Houve tendência de aumento do peso das vacas Nelore com o passar dos anos (414 kg para 1986 e $477 \mathrm{~kg}$ para 1994). Além do fator clima, inerente ao ano de parto, a maior disponibilidade de forragem, com a melhoria das pastagens e, provavelmente, o envelhecimento das vacas, apesar do efeito da idade da vaca estar incluído no modelo, são responsáveis pelos efeitos de ano sobre o peso ao parto das vacas Nelore. Para as vacas $1 / 2$ Canchim $+1 / 2$ Nelore, não houve tendência lógica de aumento ou redução no peso ao parto com o passar dos anos. Estas vacas, apesar de passarem pelos mesmos efeitos inerentes ao ano, tiveram distribuição mais equilibrada de idades dentro de ano, apresentando, portanto, menor variação de peso com o ano do parto (486 e $512 \mathrm{~kg}$, respectivamente, para 1997 e 1996). PÁDUA et al. (1994), ALENCAR et al. (1997a) e ALENCAR et al. (1999), com animais da raça Nelore e cruzados Charolês x Nelore e Limousin $x$ Nelore, também verificaram efeitos do ano de parto sobre o peso das vacas ao parto.

$\mathrm{O}$ mês do parto apresentou efeito significativo $(\mathrm{P}<0,01)$ sobre o peso ao parto de ambos os tipos de vacas (Tabela 1). As vacas paridas de agosto a novembro foram mais leves que as paridas em dezembro e de maio a julho. Estes resultados eram esperados, uma vez que as vacas paridas mais no início e no final do ano passaram o final da gestação em época de pastagens fartas. Os resultados obtidos neste trabalho estão de acordo com os obtidos por outros autores em bovinos de corte criados a pasto. BARBOSA (1986), na raça Canchim, observou maior peso ao parto para as vacas paridas no verão e outono em relação às paridas no inverno e primavera. PÁDUA et al. (1994), na raça Nelore, verificaram maior peso para as vacas paridas de março a junho, comparadas com as paridas de julho a dezembro. ALENCAR et al. (1997a, 1999) constataram maior peso ao parto para as vacas paridas durante o primeiro semestre.

A idade da vaca ao parto também apresentou efeito significativo $(\mathrm{P}<0,01)$ sobre o peso ao parto das vacas Nelore e 1/2 Canchim + 1/2 Nelore (Tabela 1 ). 
ALENCAR et al.

Tabela 1 - Análises de variância do peso da vaca ao parto (PVP) e do período de gestação (PG) das vacas Nelore e 1/2 Canchim + 1/2 Nelore

Table 1 - Analyses of variance for cow weight at calving (CWC) and gestation length (GL) for the Nellore and 1/2 Canchim $+1 / 2$ Nellore cows

\begin{tabular}{|c|c|c|c|c|c|}
\hline \multirow{4}{*}{$\begin{array}{l}\text { Fonte de } \\
\text { variação } \\
\text { Source of } \\
\text { variation }\end{array}$} & \multirow{4}{*}{$\begin{array}{c}\mathrm{g} 1^{1} \\
d f\end{array}$} & \multicolumn{4}{|c|}{$\begin{array}{l}\text { Quadrado médio } \\
\text { Mean square }\end{array}$} \\
\hline & & \multicolumn{2}{|c|}{$\begin{array}{l}\text { Nelore } \\
\text { Nellore }\end{array}$} & \multicolumn{2}{|c|}{$\begin{array}{l}\text { 1/2 Canchim }+1 / 2 \text { Nelore } \\
1 / 2 \text { Canchim }+1 / 2 \text { Nellore }\end{array}$} \\
\hline & & PVP & PG & PVP & PG \\
\hline & & $C W C$ & $G P$ & $C W C$ & $G P$ \\
\hline $\begin{array}{l}\text { Ano do parto } \\
\text { Calving year }\end{array}$ & $8 / 6$ & $10.179 * *$ & - & $7205^{* *}$ & - \\
\hline $\begin{array}{l}\text { Mês do parto } \\
\text { Month of calving }\end{array}$ & $7 / 6$ & $20.458 * *$ & - & $21.397 * *$ & - \\
\hline $\begin{array}{l}\text { Estação de monta } \\
\text { Breeding season }\end{array}$ & $9 / 8$ & - & 49 & - & 39 \\
\hline $\begin{array}{l}\text { Idade da vaca } \\
\text { Age of cow }\end{array}$ & $7 / 6$ & $9.812 * *$ & $140+$ & $114.522 * *$ & 13 \\
\hline $\begin{array}{l}\text { Origem da vaca } \\
\text { Cow origin }\end{array}$ & $4 /-$ & $24.519 * *$ & 27 & - & - \\
\hline $\begin{array}{l}\text { Sexo do bezerro } \\
\text { Sex of calf }\end{array}$ & $1 / 1$ & - & $414 *$ & - & 53 \\
\hline $\begin{array}{l}\text { Pai do bezerro } \\
\text { Sire of calf }\end{array}$ & $48 / 8$ & - & $97+$ & - & $273 * *$ \\
\hline Resíduo & $604 / 609$ & 1754 & & 2003 & \\
\hline Error & $370 / 479$ & & 75 & & 25 \\
\hline $\mathrm{R}^{2}(\%)$ & - & 44 & 20 & 51 & 21 \\
\hline
\end{tabular}

Para as vacas Nelore, houve aumento linear no seu peso do quarto $(413 \mathrm{~kg})$ para o décimo primeiro $(472 \mathrm{~kg})$ ano e, para as vacas cruzadas, aumento linear no seu peso do terceiro $(433 \mathrm{~kg})$ para o nono $(539 \mathrm{~kg})$ ano. Estes resultados são normais, pois os animais, à medida que envelhecem, tornam-se mais pesados. Outros autores (BARBOSA, 1986; PÁDUA et al., 1994; ALENCAR et al., 1997a; e ALENCAR et al., 1999) também verificaram aumento no peso das vacas com o aumento da idade ou da ordem de parto, em bovinos de corte.

A origem da vaca, como esperado, influenciou significativamente $(\mathrm{P}<0,01)$ o peso da vaca ao parto. Este fator inclui efeitos genéticos e do ambiente de criação dos animais, que normalmente afetam características de crescimento. O peso da vaca variou de 434 a $466 \mathrm{~kg}$, dependendo da sua origem.

As médias estimadas do peso ao parto foram iguais a $450 \pm 2$ e $502 \pm 2 \mathrm{~kg}$ para as vacas Nelore e 1/2 Canchim $+1 / 2$ Nelore, respectivamente. Estes valores são, em geral, maiores que os encontrados na literatura científica brasileira, para bovinos de corte. ALENCAR e BUGNER (1987), PÁDUA et al. (1994), ALENCAR et al. (1997a) e ALENCAR et al.(1999) verificaram valores que variaram de 403 a
$425 \mathrm{~kg}$ para vacas da raça Nelore. BARBOSA (1986) obteve o valor de $476 \mathrm{~kg}$ para vacas Canchim. ALENCAR e BUGNER (1989) encontraram o valor de $453 \mathrm{~kg}$ para fêmeas Canchim de segundo parto. ALENCAR et al. (1997a, 1999) observaram valores que variaram de 437 a $486 \mathrm{~kg}$ para animais de $1 / 4$ a 5/8 Charolês x Nelore.

O peso da vaca ao parto é característica de importância econômica em bovinos de corte, uma vez que, quanto maior é o animal, maior é a quantidade de alimento necessário para sua manutenção. Dessa maneira, nos sistemas de produção brasileiros, em que os animais são mantidos exclusivamente a pasto durante todo o ano, passando pelos rigores da estação seca, é importante que as vacas não sejam excessivamente grandes. O peso ao parto das fêmeas 1/2 Canchim $+1 / 2$ Nelore deste trabalho $(502 \mathrm{~kg}$ ) sugere que esses animais são de tamanho médio, apesar de serem filhas de vacas Nelore de tamanho maior que o observado normalmente. Portanto, estas fêmeas cruzadas podem ser incorporadas ao sistema de produção, para que se aproveite das vantagens das heteroses individual e materna.

A estação de monta, a origem da vaca e a idade da vaca ao parto não apresentaram efeito significativo 
964 Rev. bras. zootec.

sobre o período de gestação dos bezerros (Tabela 1). REYNOLDS et al. (1980) também não verificaram efeito significativo da idade da vaca ao parto sobre o período de gestação, em bovinos de corte. SILVA e PEREIRA (1986) observaram efeito significativo do ano do parto, fator semelhante à estação de monta deste trabalho, sobre o período de gestação de vacas $1 / 2$ Chianina $+1 / 2$ Zebu, mas não sobre o período de gestação de vacas zebuínas. Quanto à ordem de parto, os efeitos observados por esses autores foram inversos. ALENCAR et al. (1996) verificaram efeitos significativos do ano do parto e da idade da vaca sobre o período de gestação de vacas Nelore e cruzadas Tabapuã x Gir, quando acasaladas com touros da raça Nelore, sendo que o efeito da idade da vaca foi quadrático. WRAY et al. (1987) e SACCO et al. (1990) observaram aumento no período de gestação com o aumento da idade da vaca.

O sexo do bezerro apresentou efeito significativo sobre o período de gestação apenas dos bezerros $1 / 2$ Canchim $+1 / 2$ Nelore. Neste caso, o período de gestação com os bezerros machos $(292,0 \pm 0,7$ dias) foi mais longo do que com as fêmeas (289,9 $\pm 0,7$ dias). Outros autores também verificaram maior período de gestação para bezerros machos em comparação a fêmeas, em bovinos de corte (SILVA et al., 1979; REYNOLDS et al., 1980; SILVA e PEREIRA, 1986; WRAY et al., 1987; e SACCO et al., 1990). ALENCAR e BUGNER (1987) e ALENCAR et al. (1992a), entretanto, não observaram efeito de sexo do bezerro sobre o período de gestação de vacas das raças Canchim, Nelore e Guzerá, ao passo que ALENCAR et al. (1996) verificaram período de gestação mais longo para fêmeas em relação aos machos.

O pai do bezerro apresentou efeito significativo $(\mathrm{P}<0,01)$ sobre o período de gestação apenas dos bezerros 1/2 Charolês $+1 / 4$ Canchim $+1 / 4$ Nelore. Este efeito pode estar associado ao crescimento do bezerro na fase intra-uterina. SILVA et al. (1979), ALENCAR e BUGNER (1987) e ALENCAR et al. (1992a) também verificaram efeitos significativos de pai do bezerro sobre o período de gestação.

As médias estimadas do período de gestação foram iguais a $291,0 \pm 0,4$ e $285,7 \pm 0,2$ dias para os bezerros $1 / 2$ Canchim $+1 / 2$ Nelore e 1/2 Charolês + $1 / 4$ Canchim $+1 / 4$ Nelore, respectivamente. O valor obtido para os animais 1/2 Canchim é menor do que aqueles de 293,6 e 294,5 dias estimados por ALENCAR e BUGNER (1987) e SCARPATI (1997), respectivamente, para a raça Nelore. Entretanto, ALENCAR et al. (1996) obtiveram o valor de 287,6 dias para o período de gestação de animais Nelore, bem abaixo do valor estimado nesta pesquisa. Neste trabalho, os bezerros cruzados Canchim x Nelore foram mais pesados (ALENCAR et al., 1997b) ao nascimento que os Nelore puros. É possível que a vaca Nelore tenha algum mecanismo que expulse o bezerro antes que ele se torne excessivamente grande e passe a ser um problema durante o parto. O peso ao nascimento dos bezerros cruzados Canchim $\mathrm{x}$ Nelore filhos das vacas Nelore deste estudo foi igual a $31,0 \mathrm{~kg}$ (OLIVEIRA et al., 1994). De fato, ALENCAR

\begin{tabular}{|c|c|c|c|c|c|c|c|}
\hline \multirow{4}{*}{$\begin{array}{l}\text { Fonte de } \\
\text { variação }^{2} \\
\text { Source of } \\
\text { variation }\end{array}$} & \multirow{4}{*}{$\begin{array}{l}\mathrm{gl}^{1} \\
d f\end{array}$} & \multirow{2}{*}{\multicolumn{5}{|c|}{$\begin{array}{l}\text { Quadrado médio } \\
\text { Mean square }\end{array}$}} & \multirow[b]{3}{*}{ IPP } \\
\hline & & & & & & & \\
\hline & & PPI & IPI & PPC & IPC & PPP & \\
\hline & & $W F I$ & $A F I$ & WFCo & $A F C o$ & $W F C$ & $A F C$ \\
\hline Ano (A) & 7 & $4001 * *$ & $52.636 * *$ & $4046 * *$ & $64.877 * *$ & 2750 & $68.711 * *$ \\
\hline $\begin{array}{l}\text { Year }(Y) \\
\text { Época (E) }\end{array}$ & 1 & 208 & $91.418 * *$ & 6 & $68.448 * *$ & 4032 & $69.179 * *$ \\
\hline $\begin{array}{l}\text { Season }(S) \\
\mathrm{A} \times \mathrm{E} \\
Y \times S\end{array}$ & 7 & $2286+$ & $10.934 *$ & $2439+$ & $16.807^{*}$ & 889 & $17.661^{*}$ \\
\hline $\begin{array}{l}\text { Resíduo } \\
\text { Error } \\
\mathrm{R}^{2}(\%)\end{array}$ & & $\begin{array}{c}1141 \\
(203) \\
18\end{array}$ & $\begin{array}{c}4385 \\
(204) \\
40\end{array}$ & $\begin{array}{c}1239 \\
(189) \\
18\end{array}$ & $\begin{array}{c}7525 \\
(190) \\
34\end{array}$ & $\begin{array}{c}1765 \\
(193) \\
8\end{array}$ & $\begin{array}{c}7500 \\
(193) \\
35\end{array}$ \\
\hline
\end{tabular}

$+\mathrm{P}<0,07 ;{ }^{*} \mathrm{P}<0,05 ;{ }^{* *} \mathrm{P}<0,01$.

$1 \mathrm{GL}=$ graus de liberdade; GL do resíduo entre parênteses

1 Degrees of freedom; DF of the error in parentheses.

${ }^{2}$ Ano e época $=$ ano e época de nascimento da novilha.

${ }^{2}$ Year and season $=y e a r$ and season of birth of the heifer. 
et al. (1992a) observaram períodos de gestação de vacas guzeradas iguais a 282,8 e 293,2 dias, quando acasaladas com touros Canchim e Guzerá, respectivamente, apesar de os bezerros cruzados Canchim pesarem cerca de $5 \mathrm{~kg}$ a mais ao nascimento que os bezerros Guzerá (ALENCAR et al., 1992b). Na raça Canchim (62,5\% de Charolês), ALENCAR e BUGNER (1987) verificaram o valor de 288,7 dias para o período de gestação, o qual é maior que o obtido neste trabalho para os bezerros $1 / 2$ Charolês + 1/4 Canchim + 1/4 Nelore (65,6\% de Charolês).

As estimativas de herdabilidade do período de gestação, como característica do bezerro, foram iguais a $0,17 \pm 0,14$ e $0,69 \pm 0,31$ para os bezerros 1/2 Canchim $+1 / 2$ Nelore e $1 / 2$ Charolês $+1 / 4$ Canchim $+1 / 4$ Nelore, respectivamente. SILVA e PEREIRA (1986), utilizando o método dos quadrados mínimos, obtiveram o valor de 0,28 para a herdabilidade do período de gestação, como característica da vaca, para animais $1 / 2$ Chianina $+1 / 2$ Zebu. WRAY et al. (1987) obtiveram os valores de 0,37 e 0,09 para a herdabilidade do período de gestação como característica do bezerro e da vaca, respectivamente, para a raça Simental nos Estados Unidos. SCARPATI (1997), utilizando a metodologia da máxima verossimilhança restrita e modelo animal contendo os efeitos aleatórios aditivos direto e materno e de ambiente permanente, estimou o valor de 0,60 para a herdabilidade direta do período de gestação, como característica do bezerro, em animais da raça Nelore. Os resultados deste trabalho, apesar do alto erropadrão da estimativa de herdabilidade, indicam que há variação genética aditiva no período de gestação de bezerros 1/2 Charolês + 1/4 Canchim + 1/4 Nelore, filhos de vacas $1 / 2$ Canchim $+1 / 2$ Nelore e touros da raça Charolesa, suficiente para que haja resposta a um programa de seleção. O coeficiente de variação desta característica é, entretanto, muito baixo $(2,95$ e $1,75 \%$ para os bezerros $1 / 2$ Canchim $+1 / 2$ Nelore e 1/2 Charolês $+1 / 4$ Canchim $+1 / 4$ Nelore, respectivamente), o que certamente dificultará a seleção.

A interação ano x época de nascimento apresentou efeito significativo $(\mathrm{P}<0,05)$ sobre as idades à primeira inseminação, à primeira concepção e ao primeiro parto das novilhas $1 / 2$ Canchim $+1 / 2$ Nelore. Quanto aos pesos, a interação influenciou PPI e PPC em nível de $7 \%$ de probabilidade. Apesar de a época de nascimento ter apresentado efeito significativo $(\mathrm{P}<0,01)$ sobre as idades, houve diferença apenas nos anos de 1987 e 1992 e, nestes casos, as idades foram maiores para as novilhas nascidas de maio a agosto. Em outros anos, a diferença entre épocas foi

Tabela 3 - Médias estimadas ( \pm erros-padrão) do peso da vaca ao parto, de acordo com o mês do parto e o grupo genético da vaca

Table 3 - Least squares means ( \pm standard error) for cow weight at calving, according to the month of calving and genetic group of cow

\begin{tabular}{|c|c|c|c|c|}
\hline \multirow[b]{2}{*}{$\begin{array}{l}\text { Mês do parto } \\
\text { Month of calving }\end{array}$} & \multicolumn{2}{|c|}{$\begin{array}{l}\text { Nelore } \\
\text { Nellore }\end{array}$} & \multicolumn{2}{|c|}{$\begin{array}{l}\text { 1/2 Canchim }+1 / 2 \text { Nelore } \\
1 / 2 \text { Canchim }+1 / 2 \text { Nellore }\end{array}$} \\
\hline & $\mathrm{N}^{1}$ & $\begin{array}{c}\text { Média, kg } \\
\text { Mean }\end{array}$ & $\mathrm{N}^{1}$ & $\begin{array}{l}\text { Média, } \mathrm{kg} \\
\text { Mean }\end{array}$ \\
\hline $\begin{array}{l}\text { Maio } \\
\text { May }\end{array}$ & 46 & $487 \pm 7$ & 49 & $533 \pm 7$ \\
\hline $\begin{array}{l}\text { Junho } \\
\text { June }\end{array}$ & 84 & $459 \pm 5$ & 168 & $529 \pm 4$ \\
\hline $\begin{array}{l}\text { Julho } \\
\text { July }\end{array}$ & 116 & $452 \pm 4$ & 165 & $506 \pm 4$ \\
\hline $\begin{array}{l}\text { Agosto } \\
\text { August }\end{array}$ & 120 & $437 \pm 4$ & 112 & $498 \pm 5$ \\
\hline $\begin{array}{l}\text { Setembro } \\
\text { September }\end{array}$ & 108 & $431 \pm 4$ & 71 & $498 \pm 6$ \\
\hline $\begin{array}{l}\text { Outubro } \\
\text { October }\end{array}$ & 84 & $426 \pm 5$ & 34 & $467 \pm 9$ \\
\hline $\begin{array}{l}\text { Novembro } \\
\text { November }\end{array}$ & 35 & $447 \pm 7$ & 29 & $481 \pm 10$ \\
\hline $\begin{array}{l}\text { Dezembro } \\
\text { December }\end{array}$ & 38 & $462 \pm 8$ & - & - \\
\hline $\begin{array}{l}\text { Geral } \\
\text { Overall }\end{array}$ & 631 & $450 \pm 2$ & 628 & $502 \pm 2$ \\
\hline
\end{tabular}

${ }^{1} \mathrm{~N}=$ Número de observações.

${ }^{1} N=$ Number of observations. 
Rev. bras. zootec.

Tabela 4 - Médias estimadas ( \pm erros-padrão) do peso da vaca ao parto, de acordo com a idade e o grupo genético da vaca

Table 4 - Least squares means ( \pm standard error) for cow weight at calving, according to the age and genetic group of cow

\begin{tabular}{|c|c|c|c|c|}
\hline \multirow[b]{2}{*}{$\begin{array}{l}\text { Idade da vaca (anos) } \\
\text { Age of cow (years) }\end{array}$} & \multicolumn{2}{|c|}{$\begin{array}{l}\text { Nelore } \\
\text { Nellore }\end{array}$} & \multicolumn{2}{|c|}{$\begin{array}{l}1 / 2 \text { Canchim }+1 / 2 \text { Nelore } \\
1 / 2 \text { Canchim }+1 / 2 \text { Nellore }\end{array}$} \\
\hline & $\mathrm{N}^{1}$ & $\begin{array}{l}\text { Média, kg } \\
\text { Mean }\end{array}$ & $\mathrm{N}^{1}$ & $\begin{array}{c}\text { Média, } \mathrm{kg} \\
\text { Mean }\end{array}$ \\
\hline 3 & - & - & 159 & $433 \pm 4$ \\
\hline 4 & 66 & $413 \pm 7$ & 89 & $465 \pm 5$ \\
\hline 5 & 62 & $431 \pm 6$ & 127 & $508 \pm 4$ \\
\hline 6 & 105 & $447 \pm 5$ & 87 & $513 \pm 5$ \\
\hline 7 & 103 & $453 \pm 5$ & 69 & $529 \pm 6$ \\
\hline 8 & 87 & $458 \pm 5$ & 43 & $524 \pm 7$ \\
\hline 9 & 67 & $463 \pm 6$ & 54 & $539 \pm 7$ \\
\hline 10 & 51 & $464 \pm 7$ & - & - \\
\hline 11 & 90 & $472 \pm 8$ & - & - \\
\hline
\end{tabular}

${ }^{1} \mathrm{~N}=$ Número de observações.

${ }^{1} \mathrm{~N}=$ Number of observations.

grande, entretanto, em razão do pequeno número de observações em algumas classes de ano-época, os erros-padrão foram muito altos, fazendo com que a diferença fosse não significativa. O efeito de ano de nascimento $(\mathrm{P}<0,01)$ sobre IPI, IPC, IPP, PPI e PPC, também, dependeu da época de nascimento da novilha. Provavelmente, as diferenças nas estações de monta, bem como nas condições climáticas e de disponibilidade de forragem, de ano para ano, são responsáveis pela interação ano x época de nascimento.

Alguns autores verificaram efeitos significativos de ano e época ou mês de nascimento sobre as características estudadas neste trabalho para as novilhas (OLIVEIRA FILHO et al., 1975; B ALIEIRO et al., 1980; MIRANDA et al., 1982; e ALENCAR e BUGNER, 1986 e 1987), em bovinos de corte. Entretanto, MARIANTE (1978), na raça Nelore, e OLIVEIRA FILHO et al. (1979) e ALENCAR et al. (1982), na raça Canchim, não observaram efeito de ano e mês de nascimento sobre a idade ao primeiro parto das novilhas.

As médias estimadas obtidas foram iguais a 372 $\pm 2 \mathrm{~kg}$ (PPI), 376 $\pm 2 \mathrm{~kg}$ (PPC), 442 $\pm 3 \mathrm{~kg}$ (PPP), 810 \pm 5 dias (IPI), $831 \pm 6$ dias (IPC) e $1116 \pm 6$ dias (IPP). A idade ao primeiro parto obtida neste trabalho (36,7 meses) foi inferior aos valores que variaram de 39,4 a 47,3 meses, obtidos por OLIVEIRA FILHO et al. (1975 e 1979), MARIANTE (1978), AROEIRA e
ROSA (1982), MIRANDA et al. (1982), ALENCAR e BUGNER (1986 e 1987) e PÁDUA et al. (1994), para as raças Nelore e Canchim, concorda com o valor de 36,7 meses verificado por ABREU (1991), para o Nelore pele rosa, e é superior ao valor de 33,6 meses encontrado por ALENCAR et al. (1982), para a raça Canchim.

\section{Conclusões}

Os fatores de meio ano e mês do parto, idade da vaca ao parto e origem da vaca, são importantes fontes de variação sobre o peso da vaca ao parto e devem ser considerados por ocasião da avaliação e comparação de animais com base nesta característica.

O período de gestação de animais cruzados $1 / 2$ Charolês $+1 / 4$ Canchim $+1 / 4$ Nelore, filhos de vacas cruzadas 1/2 Canchim $+1 / 2$ Nelore, apresenta variação genética de alta magnitude, sugerindo que a seleção para esta característica deve resultar em progresso genético, o que é dificultado, entretanto, pela baixa variação fenotípica apresentada pela característica.

Fêmeas cruzadas 1/2 Canchim $+1 / 2$ Nelore podem apresentar o primeiro parto em idade jovem, mesmo quando criadas em regime exclusivo de pastos, e não são excessivamente grandes quando adultas, podendo ser aproveitadas nos sistemas de produção de carne bovina. 


\section{Referências Bibliográficas}

ABREU, U.G.P. Avaliação genética quantitativa de caracteres reprodutivos de um rebanho Nelore, variedade pele rosa. Jaboticabal, SP: Faculdade de Ciências Agrárias e Veterinárias, UNESP. 1991. 116p. Dissertação (Mestrado) - Faculdade de Ciências Agrárias e Veterinárias, Universidade Estadual de São Paulo, 1991.

ALENCAR, M.M., BARBOSA, R.T., BARBOSA, P.F. et al. Duração do período de gestação de vacas guzerás acasaladas com touros das raças Guzerá e Canchim, na região de Governador Valadares, MG. In: REUNIÃO ANUAL DA SOCIEDADE BRASILEIRA DE ZOOTECNIA, 29, 1992, Lavras. Anais...Lavras: SBZ, 1992a, p.221.

ALENCAR, M.M., BARBOSA, P.F., BARBOSA, R.T. et al. Desenvolvimento de bezerros guzerás e cruzados Canchim $\mathrm{x}$ Guzerá na região de Governador Valadares, MG. In: REUNIÃO ANUAL DA SOCIEDADE BRASILEIRA DE ZOOTECNIA, 29, 1992, Lavras. Anais...Lavras: SBZ, 1992b, p.202.

ALENCAR, M.M., BARBOSA, P.F., TREMATORE, R.L. et al. Peso ao parto, período de gestação e desempenho produtivo de vacas da raça Nelore e cruzadas Tabapuã x Gir. In: REUNIÃO ANUAL DA SOCIEDADE BRASILEIRA DE ZOOTECNIA, 33, 1996, Fortaleza. Anais...Fortaleza: SBZ, 1996, p.130-132.

ALENCAR, M.M., BEOLCHI, E.A., COSTA, J.L. et al. 1982. Herdabilidade da idade ao primeiro parto de vacas da raça Canchim. Pesq. Agropec. Bras., 17(8):1233-1236.

ALENCAR, M.M., BUGNER, M. 1986. Estudo da idade ao primeiro parto de vacas da raça Canchim. R. Soc. Bras. Zootec., 15(2):151-156.

ALENCAR, M.M., BUGNER, M. 1987. Desempenho produtivo de fêmeas das raças Canchim e Nelore. II. Primeiro parto. Pesq. Agropec. Bras., 22(8):867-872.

ALENCAR, M.M., BUGNER, M. 1989. Desempenho produtivo de fêmeas das raças Canchim e Nelore. IV. Segundo parto. Pesq. Agropec. Bras., 24(10):1217-1220.

ALENCAR, M.M., OLIVEIRA, J.A.L., ALMEIDA, M.A. 1999. Idade ao primeiro parto, peso ao parto e desempenho produtivo de vacas nelores e cruzadas Charolês x Nelore. Rev. bras. zootec., 28(4):681-686.

ALENCAR, M.M., TREMATORE, R.L., BARBOSA, P.F. et al. 1997b. Desempenho de bezerros filhos de touros das raças Nelore e Canchim e cruzados $\left(\mathrm{F}_{1}\right)$ Charolês x Nelore e Piemontês x Nelore. R. Bras. Zootec., 26(3):461-466.

ALENCAR, M.M., TREMATORE, R.L., OLIVEIRA, J.A.L. et al. 1997a. Desempenho produtivo de vacas da raça Nelore e cruzadas Charolês x Nelore, Limousin x Nelore e Tabapuã x Gir. R. Bras. Zootec., 26(3):467-472.

AROEIRA, J.A.D.C., ROSA, A.N. 1982. Desempenho reprodutivo de um rebanho Nelore. Pesq. Agropec. Bras., 17(2):337-343.

BALIEIRO, E.S., CARNEIRO, G.G., SILVA, H.M. et al. Herança e meio como causas de variação da idade à primeira fecundação em vacas da raça Guzerá. In: REUNIÃO ANUAL DA SOCIEDADE BRASILEIRA DE ZOOTECNIA, 17, Fortaleza. Anais... Fortaleza: SBZ, 1980, p.137.

BARBOSA, P.F. Influência de fatores genéticos e de ambiente no peso ao parto de vacas da raça Canchim. In: REUNIÃO ANUAL DA SOCIEDADE BRASILEIRA DE ZOOTECNIA, 23, 1986, Campo Grande. Anais...Campo Grande: SBZ, 1986, p.305.

BOURDON, R.M., BRINKS, J.S. 1983. Calving date versus calving interval as a reproductive measure in beef cattle. $J$. Anim. Sci., 57(6):1412-1417.

DICKERSON, G. 1969. Experimental approaches in utilizing breed resources. Animal breeding Abstracts, 37: 191-202.

EUCLIDES FILHO, K., FIGUEIREDO, G.R., EUCLIDES, V.P. 1995. Eficiência de produção de vacas de corte com diferentes potenciais para produção de leite. Pesq. Agropec. Bras., 30(7):1003-1007.

MARIANTE, A.S. Growth and reproduction in Nelore cattle in Brazil: Genetic parameters and effects of environmental factors. Gainesville: University of Florida, 1978. 131p. Tese (Phylosophy Doctor) - University of Florida, 1978.

McMORRIS, M.R., WILTON, J.W. 1986. Breeding systems, cow weight and milk yield effects on various biological variables in beef reproduction. J. Anim. Sci., 63(5):1361-1372.

MIRANDA, J.J.F., CARNEIRO, G.G., PENNA, V.M. et al. 1982. Estudo dos fatores ambientais relacionados com a idade à primeira cria na raça Nelore. Arq. Esc. Vet. UFMG, 34(2): 375-380.

OLIVEIRA, A.F., BARBOUR, J.J., ALENCAR, M.M. et al. Influência de fatores genéticos e do meio sobre o desenvolvimento de bezerros cruzados Canchim x Nelore. REUNIÃO ANUAL DA SOCIEDADE BRASILEIRA DEZOOTECNIA, 31, 1994, Maringá. Anais...Maringá: SBZ, 1994, p.153.

OLIVEIRA FILHO, E.B., DUARTE, F.A.M., KOGER, M. 1979. Genetic effects on reproduction in Canchim cattle. $R$. Bras. Genet., 2(4):281-293.

OLIVEIRA FILHO, E.B., MOREIRA, H.A., CARNEIRO, G.G. et al. 1975. Idade à primeira cria em um rebanho Nelore. Arq. Esc. Vet. UFMG, 27(2):141-153.

PÁDUA, J.T., MUNARI, D.P., WATANABE, Y.F. et al. 1994. Avaliação de efeitos de ambiente e da repetibilidade de características reprodutivas em bovinos da raça Nelore. $R$. Soc. Bras. Zootec., 23(1):126-132.

REYNOLDS, W.L., DeROUEN, T.M., MOIN, S. et al. 1980. Factors influencing gestation length, birth weight and calf survival of Angus, Zebu and Zebu cross beef cattle. J. Anim. Sci., 51(4): 860-867.

SACCO, R.E., BAKER, J.F., CARTWRIGHT, T.C. et al. 1990. Measurements at calving for straightbred and crossbred cows of diverse types. J. Anim. Sci., 68(10):3103- 3108.

SAS statistical analysis systems user's guide. 1996. Version 6.12 ed. Cary: SAS Institute.

SCARPATI, M.T.V. Modelos animais alternativos para estimação de componentes de (co)variância e de parâmetros genéticos e fenotípicos do período de gestação na raça Nelore. Ribeirão Preto, Faculdade de Medicina de Ribeirão Preto, 1997.71p. Dissertação (Mestrado em Ciências)- Universidade de São Paulo, 1997.

SILVA, H.M., SAMPAIO, I.B.M., VILLALBA, J.J.C. 1979. Reprodução em gado Nelore. I. Idade à primeira fecundação e período de gestação em Chianino - Nelore. Arq. Esc. Vet. $U F M G, 31(3): 443-450$.

SILVA, M.A., PEREIRA, F.A. 1986. Fatores de meio e genéticos que influem no desempenho reprodutivo de fêmeas Zebu e mestiças Chianina-Zebu. R. Soc. Bras. Zootec., 15(2):132-141.

SWIGER, L.A., HARVEY, W.R., EVERSON,D.U. et al. 1964. The variance of intraclass correlation involving groups with one observation. Biometrics, 20:818-826.

WILLHAM, R.L. Purebreending: achieving objectives. In: BREEDING FOR BEEF, Peebles, 1971. Proceedings... Peebles, 1971, v.1, p.15-21.

WRAY, N.R., QUAAS, R.L., POLLAK, E.J. 1987. Analysis of gestation length in american Simmental cattle. J. Anim. Sci., 65(4):970-974.

Recebido em: 12/02/98 Aceito em: 15/03/99 